2019-01-31

\title{
Impact of Time Pressure on Dentists' Diagnostic Performance
}

\author{
Plessas, Anastasios
}

http://hdl.handle.net/10026.1/13270

10.1016/j.jdent.2019.01.011

Journal of Dentistry

Elsevier

All content in PEARL is protected by copyright law. Author manuscripts are made available in accordance with publisher policies. Please cite only the published version using the details provided on the item record or document. In the absence of an open licence (e.g. Creative Commons), permissions for further reuse of content should be sought from the publisher or author. 


\section{Accepted Manuscript}

Title: Impact of Time Pressure on Dentists' Diagnostic Performance

Authors: Anastasios Plessas, Mona Nasser, Yaniv Hanoch, Timothy O'Brien, Maria Bernardes Delgado, David Moles

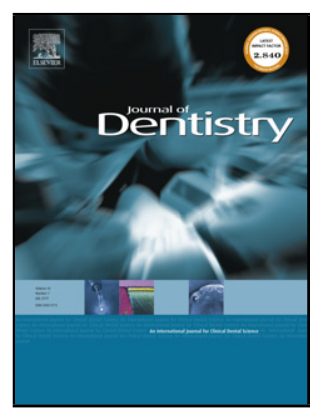

PII:

S0300-5712(19)30020-X

DOI: https://doi.org/10.1016/j.jdent.2019.01.011

Reference: JJOD 3082

To appear in: Journal of Dentistry

Received date: $\quad 11$ December 2018

Revised date: 11 January 2019

Accepted date: $\quad 14$ January 2019

Please cite this article as: Plessas A, Nasser M, Hanoch Y, O'Brien T, Bernardes Delgado M, Moles D, Impact of Time Pressure on Dentists' Diagnostic Performance, Journal of Dentistry (2019), https://doi.org/10.1016/j.jdent.2019.01.011

This is a PDF file of an unedited manuscript that has been accepted for publication. As a service to our customers we are providing this early version of the manuscript. The manuscript will undergo copyediting, typesetting, and review of the resulting proof before it is published in its final form. Please note that during the production process errors may be discovered which could affect the content, and all legal disclaimers that apply to the journal pertain. 
Title: Impact of Time Pressure on Dentists' Diagnostic Performance.

Short Title: Time pressure and dentists' performance

Mr Anastasios* Plessasaanastasios.plessas@plymouth.ac.uk , Dr Mona Nasseramona.nasser@plymouth.ac.uk , Professor Yaniv Hanochbyaniv.hanoch@plymouth.ac.uk , Mr Timothy O'Brien ${ }^{a}$ timothy.obrien@plymouth.ac.uk , Miss Maria Bernardes Delgadoa mariadocarmo.bernardesdelgado@plymouth.ac.uk , Professor David Molesadavid.moles@plymouth.ac.uk,

Mr Anastasios Plessas ${ }^{a}$, NIHR Academic Clinical Fellow in General Dental Practice

Dr Mona Nassera, Associate Professor of Evidence Based Dentistry

Professor Yaniv Hanoch ${ }^{\mathrm{b}}$, Professor of Decision Science

Mr Timothy O’Briena, Clinical Associate Professor in Restorative Dentistry

Miss Maria Bernardes Delgadoa, NIHR Academic Clinical Fellow in General Dental Practice

Professor David Moles ${ }^{a}$, Professor of Oral Health Services Research

aPeninsula Dental School, Faculty of Medicine and Dentistry, University of Plymouth, Plymouth, PL6 8BT, England, UK

anastasios.plessas@plymouth.ac.uk

mona.nasser@plymouth.ac.uk

timothy.obrien@plymouth.ac.uk

mariadocarmo.bernardesdelgado@plymouth.ac.uk

david.moles@plymouth.ac.uk

bSchool of Psychology, Faculty of Health and Human Sciences, University of Plymouth, Plymouth, PL4 8AA, England, UK 
yaniv.hanoch@plymouth.ac.uk

Corresponding author: Mr Anastasios Plessas (anastasios.plessas@plymouth.ac.uk ) Peninsula Dental School, Faculty of Medicine and Dentistry, University of Plymouth, Plymouth, PL6 8BT, England, UK

ORCID: 0000-0002-6360-8895

\begin{abstract}
:
Objectives: Although stress is prevalent amongst dentists, there is a paucity of data on the impact of stressors on dentists' clinical performance. To address this gap in the literature, the aim of the present study was to explore the role of time pressure, representing one common stressor, on dentists' radiographic diagnostic performance.
\end{abstract}

Methods: Forty dentists were randomised to examine and provide a radiographic report on two sets of radiographs (six bitewings in each set) under two conditions on a cross-over basis: time-pressure vs. no-time-pressure. The radiographic report of an experienced consultant was considered the gold standard against which participants diagnostic decisions were compared to calculate sensitivity and specificity. Participants rated their stress after each experimental condition using a $100 \mathrm{~mm}$ visual analogue scale (VAS).

Results: The VAS scores for stress were significantly higher in the time pressure condition vs. no time pressure condition (mean: 55.78 versus 10.73, $\mathrm{p}<0.0001$ ), indicating that the time pressure acted as a source of stress. Dentists' diagnostic performance was affected; the sensitivity was significantly lower under time pressure (median: 0.50 versus $0.80, p<0.0001$ ), but by contrast, the median diagnostic specificity was 1.00 under both conditions. 
Conclusions: Time pressure negatively impacts one aspect of dentists' diagnostic performance, namely sensitivity (increased diagnostic errors and omissions of pathology), which can potentially affect patient safety and the quality of care delivered. However, time pressure was found to have less influence on diagnostic specificity.

Clinical Significance: The present study demonstrated a significant deterioration of dentists' diagnostic performance (sensitivity) under time-pressure when examining bitewing radiographs. Diagnostic errors may put patient safety at risk, with patients potentially being harmed if pathology is missed. Such errors can have medicolegal implications on the dentists' practice.

Keywords: 1) Radiography, Bitewing; 2) Stress, Psychological; 3)Diagnostic Errors; 4) Dental Caries; 5) Alveolar Bone Loss; 6) General Practice, Dental.

\section{Introduction}

Dentistry is recognised as a stressful profession [1-3]. Work related stress has been associated with dentists' developing mental health issues such as depression and an elevated risk of burnout [4]. The prevalence of burnout amongst the profession varies amongst studies with some reporting that $2.5 \%$ of the dental workforce is severely burnt-out [5] and others indicating that $26 \%$ of the dentists are at high risk of both emotional exhaustion and depersonalisation [6].

Dental professionals encounter numerous sources of stress beginning in dental school [7] and postgraduate training [8] that can continue or escalate during their practicing 
lives. The most often reported stressors are dealing with difficult and demanding patients, running behind schedule and time pressures, followed by staff issues and pressures from third parties [1, 9-15]. Although dentists perceive time pressure as an important stressor which may potentially influence their clinical decisions [16, 17], a systematic review of the dental literature identified no experimental studies that assessed the impact of stress or different stressors on dentists' performance [18]. In contrast, there is ample evidence from other medical domains showing that time pressure affects decision making and diagnostic accuracy of healthcare professionals as well as their psychomotor performance and procedural skills [19-23]. Whether time pressure has similar impact on dentists' performance, however, is an open and important question.

Dental complaints and negligence claims have been reported to be on the rise [24], with $9 \%$ of the dental claims relating to diagnostic errors [25], whilst, incorrect interpretation of diagnostic tests account for $37 \%$ of medical claims [26]. Bitewing radiographs are the most common radiograph utilised in general practice to assist the dentist to form a diagnosis and inform their treatment decisions [27-29]. Bitewings are often taken at relatively regular intervals, whenever there is a suspicion of dental caries and/or periodontal disease $[29,30]$. If time pressure has a negative impact on dentists' diagnostic ability, this may result in sub-optimal care being provided and potentially put patient safety at risk.

Therefore, given the paucity of research in this topic, the aim of this paper was to investigate the impact of time pressure on dentists' diagnostic performance when viewing dental bitewing radiographs.

\section{Material and Methods}




\section{Design}

This was a randomised cross-over study. Primary care dentists examined and provided a radiographic report on two sets of radiographs A and B (six bitewings in each set) under two conditions: time-pressure vs. no-time-pressure. Dentists were randomised to one of four groups based on the order in which they examined the two sets of radiographs ( $A$ then $B$, or $B$ then $A$ ) and the order in which the examination conditions were applied (time pressure then no time pressure, or no time pressure then time pressure). The radiographic report of an experienced consultant was considered the gold standard against which participants diagnostic decisions were compared to calculate sensitivity and specificity. The study received ethical approval (16/17-704) from the University of Plymouth Research Ethics committee, England, UK.

\section{Recruitment}

Primary care dentists in the Southwest of England were invited to participate in the study. Invitation leaflets and information sheets were sent to potential participants via post, or emails through different professional dental networks.

\section{Radiographs}

Twelve digital radiographs were selected from the Peninsula Dental Social Enterprise's clinical patient records, to develop two sets of dental bitewing radiographs (A and B). Each set was composed of six radiographs. Between the two sets of radiographs, the radiographs' assessed diagnostic difficulty, the number of pathological features, and the types of different pathologies were distributed evenly. Each set was composed of three easy and three difficult radiographs. The level of 
difficulty was established via a pilot study using six experienced (8-20 years) dentists. The number and type of pathological features were determined by a specialist in restorative dentistry (TO'B) who provided a detailed radiographic report. The report was also reviewed by a general dentist (AP). This report was used as gold standard radiographic report.

\section{Time Pressure}

The observation time for each of the twelve radiographs in the time pressure condition was determined by the pilot study, using the equation suggested by Ordonez and Benson: TP=TMP-SDP [31]. TMP corresponded to the mean observation time for each radiograph in the pilot study, while SDP to the standard deviation for each radiograph in the pilot study. The time pressure limits applied on the examination of the study radiographs varied from 8 to 38 seconds.

\section{Experimental Groups}

Four different counterbalanced experimental test groups were developed on Qualtrics Research CORE ${ }^{\text {TM }}$ interface and a web link was generated for each group (see Figure 1). Block randomisation was used to ensure that equal numbers of participants were allocated in each of the four experimental groups [32]. STATA version 10 was used for the random sequence generation and the size of blocks varied between 4 and 8 . This stage was completed by an independent researcher (DM) and the sequence and size of blocks remained unknown to the principal researcher (AP).

\section{Procedure}

The study took place in either a quiet non-clinical room on the premises of the University of Plymouth or individual dental practices during non-operating hours. The 
study was delivered electronically on a computer and took on average forty minutes to complete.

After informing the participants about the procedure, a written consent form was signed and the participants were allocated to one of the four groups. The group they were allocated to was revealed after opening a sealed envelope. The corresponding Qualtrics web link then was opened on the computer. Prior to commencing the study, the participants undertook a practice exercise which mimicked the experimental condition. Next, participants were instructed to examine each radiograph and to take notes on a blank piece of paper (pen and paper were provided to all participants). In the time pressure condition, a countdown clock was present on the screen (Figure. 2). After viewing each radiograph they were asked to provide a report on any pathology they identified and be tooth and site specific. To reduce variations of reporting style or terminology among participants, participants were asked to indicate the types of pathology they identified, based on a list that was provided to them (Table 1). Although the examination of the radiographs was subject to time pressure on half of all occasions, there was no time restriction applied to the participants while they typed the radiographic reports for either of the conditions. After each condition, the participants were asked to complete two $100 \mathrm{~mm}$ electronic visual analogue scales (100 mm VAS) indicating the level of stress they experienced during the task and their perception of the difficulty of the task (Figure 3). At the end of the study, participants completed a number of demographics questions.

Data were extracted from each radiographic report by the principal researcher (AP). The completed data collection forms were double-checked by another researcher (MBD) against the participants' reports to ensure that no transcription errors had occurred. Each participant's responses were compared to the gold-standard 
radiographic report. For each report the true-positive (TP), true-negative (TN), falsepositive (FP) and false negative (FN) decisions were calculated. For each radiographic report the participant's diagnostic sensitivity and specificity were calculated according to the following diagnostic equations: Sensitivity $=T P / T P+F N$, and Specificity $=F P /$ $\mathrm{FP}+\mathrm{TN}[33]$.

\section{Statistical analysis}

All analyses were performed using SPSS version 24 (IBM SPSS Statistics) and the significance level was set at $p<0.05$. The difference between VAS values (selfreported stress and difficulty) between the two conditions were analysed using paired $t$-tests. Mann-Whitney $U$ tests were used to assess the difference between the diagnostic sensitivity and specificity between the two conditions for all the types of pathology and for caries and bone loss recognition alone. The analyses were performed by condition globally across all the study radiographs and also by individual radiograph.

\section{Results}

\section{Participants}

Forty general dental practitioners from the Southwest of England took part in the study.

Their experience ranged from 1 to 35 years with a mean of 17 years $(16.92 \pm 12.58$ (SD)years). The participant characteristics are depicted in Table 2.

\section{Stress and perceived difficulty of the task}

To examine whether the time pressure manipulation worked, participants' stress and perceptions of the difficulty of the task were compared between the two conditions. The data showed a statistically significant difference $(p<0.001)$ in the VAS scores for 
perceived stress under the time-pressure (Mean $=55.78, S D=25.74$ ) compared to the no time-pressure (Mean $=10.73, S D=12.06)$ condition. Similarly, participants rated the experimental task significantly more difficult $(p<0.001)$ under the time pressure condition (Mean=65.43, SD=25.11), compared to the no time pressure condition (Mean=14.83, SD=12.63).

\section{Impact of time pressure on diagnostic performance in global radiographic} reporting (all pathologies and features)

To evaluate the impact of time pressure on performance, we compared diagnostic results under the two conditions globally. A Mann Whitney $U$ test indicated that median diagnostic sensitivity was significantly worse $(p<0.001)$ under time pressure (Median=0.50) compared to under no time pressure (Median=0.80), for all radiographs combined. Further, Cohen's effect size value $(r=0.41)$ suggested a moderate effect of time pressure on the dentists' diagnostic ability. A statistically significant $(p=0.036)$ albeit negligible effect $(r=0.07)$ of time pressure on dentists' diagnostic specificity was observed. The median value for specificity for both conditions was 1.00 .

\section{Impact of time pressure on radiographic caries recognition.}

A Mann Whitney U test indicated that participants' global ability to correctly diagnose lesions indicative of caries radiographically (sensitivity) was significantly worse $(p<0.001)$ under time pressure (Median=0.6) compared to no time pressure (Median=1). Cohen's effect size value $(r=0.29)$ suggested a moderate effect of time pressure on dentists' diagnostic ability. In contrast, the dentists' ability to correctly identify caries free sites radiographically, did not appear to be affected by time pressure (Median for both conditions 1.00, $\mathrm{p}=0.443$ ). 


\section{Impact of time pressure on radiographic bone loss recognition.}

We also evaluated whether time pressure negatively affected participants' ability to recognise and report on radiographic evidence of bone loss. In particular, the participants were asked to report on radiolucencies indicative of horizontal, vertical and furcation bone loss. A Mann Whitney $U$ test indicated that participants' global ability to correctly diagnose bone loss and bony defects radiographically (sensitivity) was significantly worse $(p<0.001)$ under time pressure $($ Median=0.33) compared to no time pressure (Median=1.00). Cohen's effect size value $(r=0.35)$ suggested a moderate effect of time pressure on dentists' diagnostic ability. In contrast, dentists diagnostic specificity did not appear to be affected by time pressure (Median for both conditions $1.00, p=0.353)$.

\section{Impact of time pressure on dentists' diagnostic performance for each radiograph.}

The impact of time pressure on diagnostic performance was compared for each of the 12 different radiographs separately to check whether the results were consistent across all radiographs (Tables 3 and 4). The participants' median diagnostic sensitivity was significantly worse for 10 out of 12 of the radiographs under the time pressure condition, but their median diagnostic specificity was not significantly different between the experimental conditions for any of the radiographs. The median sensitivity values varied from 0.21 to 1.00 for the time-pressure condition, and from 0.50 to 1.00 for the non-time-pressure condition. The median specificity was high in both conditions ranging from 0.98 to 1.00 across all the radiographs.

When focusing on caries recognition, in contrast, in only two (A4 and B1) of the radiographs did the median diagnostic sensitivity of the dentists deteriorate under time 
pressure statistically significantly (by $50 \%$ and $10 \%$ respectively). In the reports of six radiographs no statistically significant difference between dentists median diagnostic sensitivity was observed in relation to time pressure. The sensitivity could not be calculated for the reports of four radiographs as there was no caries-related pathology present. For all the radiographic reports the median specificity was high (Median ranging from 0.91 to 1.00 ) and no statistically significant differences were detected between the conditions.

For bone loss recognition, the participants' median diagnostic sensitivity was significantly worse for 6 out of 9 of the radiographs under the time pressure condition. The median sensitivity values varied from 0.33 to 1.00 for the time-pressure condition, and from 0.50 to 1.00 for the non-time-pressure condition. The sensitivity could not be calculated for the reports of three radiographs as there was no bone loss present. Lastly, the median specificity did not differ significantly for either condition and it was 1.00 for all the radiographs.

\section{Discussion}

Time pressures occur frequently in general dental practice and dentists have reported that time constraints may influence the decisions they make in their day to day practice $[11,17]$. This study examined the influence of time pressure on the dentists' diagnostic performance when examining dental bitewing radiographs in a simulated environment. To the best of our knowledge, this is the first study to empirically examine the impact of time pressure on dentists' performance [18].

Our data clearly reveal a significant deterioration in dentists' diagnostic sensitivity both in terms of identifying all the reporting items in the given radiographs $(30 \%$ median 
reduction in sensitivity), recognising radiolucencies that are indicative of caries (40\% median reduction in sensitivity), and recognising radiographic signs of periodontal destruction (67\% median reduction in sensitivity). However, no clinically relevant differences were observed for changes in specificity.

It has been proposed that when dentists examine dental bitewing radiographs, they use a 'script match' based on cumulative previous experience with similar clinical presentations of health and disease [34]. This script match is known as pattern recognition [34]. Our findings partially support the dual process theory of decision making which suggests that two distinct psychological processes are at work when a clinician is diagnosing a case: System 1 non-analytical reasoning and System 2 analytical reasoning $[35,36]$. Non-analytical reasoning, also called heuristic reasoning, depends on rapid, unconscious pattern recognition during which prior examples or illness scripts stored in long-term memory are retrieved. This type of reasoning is quick, intuitive, implicit, contextualized, and typically efficient in diagnosing routine cases [35, 36]. Thus, dentists may employ System 1 when examining radiographs, but its efficiency may be impaired under time pressure and become vulnerable to errors. On the other hand, System 2 reasoning is slow, reflective, sequential, effortful, and particularly used to diagnose complex cases $[35,36]$. Dentists, therefore, may employ System 2 reasoning when collating information from the patient's history, symptoms and clinical presentation to come up with a definite clinical diagnosis.

Our findings are also in line with previous studies indicating a negative impact of time pressure on diagnostic performance among other groups of health care professionals. A randomised controlled experiment by ALQahtani et al., for example, reported that internal medicine residents made on average $37 \%$ more diagnostic errors under time pressure than their peers in the control no-time-pressure group [22]. Similarly, in a 
simulated study by Tsiga et al., primary care medical practitioners gave significantly more incorrect responses when they answered questions related to diagnosis and management of different respiratory infection clinical scenarios under time pressure than under no time constrains [23].

Time pressures faced in general dental practice, therefore, may have a negative impact on the quality of care delivered. Diagnostic errors may put patient safety at risk, with patients potentially being harmed if pathology is missed. This may lead to unnecessary pain, the need for more complex treatment such as endodontic treatment, or even loss of the tooth. Besides, bitewing radiographs offer important diagnostic information with regard to alveolar bone destruction allowing the practitioner to detect and monitor any deterioration in periodontal health and support [37]. Medico-legal defence unions report an increasing number of claims related to allegations of failure to diagnose and treat periodontitis $[38,39]$. Our study indicated that time pressure may lead to failure to identify and report radiographic signs of caries and periodontal disease (such as vertical and furcation bone loss) which may in turn have detrimental medico-legal implications on the dentists' practice.

Allowing longer appointment times when dentists are planning to take and report on radiographs and/or re-assessing and reviewing their radiographs and radiographic reports when they are not under time constraints may mitigate the risk of diagnostic errors, but would naturally have resource implications. Therefore, changes in dental guidelines, policy and regulation might be warranted to facilitate longer appointment times. However, this would be difficult to implement since policy-makers and regulators do not specify how long appointments should take. Practitioners decide on the length of time required to undertake dental appointments and this decision represents a complex interplay between how long the practitioner feels is necessary 
to undertake the task and the efficiency (and therefore cost/profit) that they are attempting to achieve. Further evidence on the role of time pressure on dentists' efficiency, patient experience and patient safety is required in order to inform the debate and influence policy makers' decisions [40].

\section{Strengths and limitations}

In our study, participating dentists were working in a variety of primary care settings (private, NHS, mixed practices and community services) and had a broad range of experience (1-35 years) which increases the generalisability of the findings of this study. The counterbalancing and randomisation procedures ensured that the complexity and the order of the tasks were balanced and unaffected by participants' learning or by any potential fatigue by the end of the study. Thus, the observed difference in diagnostic performance is most likely primarily due to the experimental manipulation (time pressure). Moreover, the time pressure limits applied were calculated from a pilot study based on previously validated methods [31]. The statistically significant difference in VAS scores between the two conditions confirmed that the time pressure in the present study acted as a stressor factor amongst the participants.

Whether the time pressure limits used in the present study reflect the time constrains that dentists may face in clinical practice is an open question as currently we are lacking such data from general dental practice. In addition, the simulated nature of the experimental task does not represent fully what happens in a real clinical environment. Dentists in practice may face more than one stressor simultaneously (time pressure, demanding patient, difficult treatment). These are conditions which are difficult to replicate in a simulated experimental setting. Stressful encounters with real 
patients, however, are difficult to predict and raise ethical concerns if manipulated in the real setting. Nonetheless, despite these limitations, our study provides a vital indication regarding the likely impact of time pressure on dentists' performance.

\section{Implications for Future Research}

Future studies can build upon the present study by using different types of radiographs and accompany each radiograph or set of radiographs with a clinical scenario (e.g. vignette, clinical photographs). The use of adjunctive clinical information or scenarios in future studies would allow exploration of how different priming information may affect dentists' diagnostic decisions and increase the applicability of the findings to clinical practice. The deterioration in sensitivity, but not specificity is consistent with practitioners missing information, rather than misinterpreting information. However, whilst we have observed these changes, the mechanisms that underpin them remain hypothetical and need to be explored empirically. Eye-tracking technology, being widely used in psychology and human factors research, can be used to assess the dentists' information processing strategies under time pressure versus no-time pressure. [41]. Employing eye tracking technology in future studies may give a better insight into the potential underlying mechanisms (cognitive or behavioural) explaining any observed difference in performance between the two conditions. Understanding these mechanisms may also facilitate and inform the development of tools to decrease the risk of diagnostic errors, such as decision support systems.

Given the paucity of experimental research on this topic, future studies are also warranted to evaluate the impact of time pressure on other aspects of dentists' performance such as treatment planning, communication and psychomotor skills. These research findings could inform the future development of appropriate 
educational, patient safety or quality improvement interventions to address performance issues.

\section{Conclusion}

The present experimental study demonstrated that when examining dental bitewing radiographs in a simulated environment, dentists missed pathological features (sensitivity). The deterioration in sensitivity (30\% for all pathological features, $40 \%$ for caries related pathology and $67 \%$ for periodontal disease related pathology) was not only statistically but also clinically significant. However, dentists' ability to correctly identify sound sites and teeth free of pathology (specificity) was not materially affected.

Declarations of Interest: The authors declare no conflict of interest. Mr Anastasios Plessas and Miss Maria Bernardes Delgado are NIHR (National Institute for Health Research) funded Academic Clinical Fellows.

\section{Acknowledgements}

The authors would like to thank Mr Robert Witton for granting access to the Peninsula Dental Social Enterprise patient records. Also the secretaries of: Peninsula Dental Social Enterprise, Plymouth British Dental Association section, Exeter British Dental Association section, South West Local Dental Committees (LDC ), South West Denplan Sections, Faculty of General Dental Practice (FGDP) and British Association for the Study of Community Dentistry (BASCD) for disseminating the study invitations through email. We would like to thank the dentists who participated in the pilot study and the dentists who gave up their spare time to participate in the study. The authors declare no conflict of interest. Mr Anastasios Plessas and Miss Maria Bernardes 
Delgado are NIHR (National Institute for Health Research) funded Academic Clinical Fellows. 


\section{References}

[1] H.L. Myers, L.B. Myers, 'It's difficult being a dentist': stress and health in the general dental practitioner, Br. Dent. J. 197(2) (2004) 89-93; discussion 83; quiz 100-1.

[2] C. Wilks, Occupational stress in dentistry, Br. Dent. J. 179(1) (1995) 9.

[3] R.F. Wilson, P.Y. Coward, J. Capewell, T.L. Laidler, A.C. Rigby, T.J. Shaw, x’, Br. Dent. J. 184(10) (1998) 499-502.

[4] J. Larbie, M. Kemp, P. Whitehead, The Mental Health and Well-being of UK Dentists: A Qualitative Study, BDA, London, 2017.

[5] R.C. Gorter, G. Albrecht, J. Hoogstraten, M.A. Eijkman, Professional burnout among Dutch dentists, Community Dent. Oral Epidemiol. 27(2) (1999) 109-16.

[6] R.C. Gorter, R. Freeman, Burnout and engagement in relation with job demands and resources among dental staff in Northern Ireland, Community Dent. Oral Epidemiol. 39(1) (2011) 87-95.

[7] A.M. Alzahem, H.T. van der Molen, A.H. Alaujan, H.G. Schmidt, M.H. Zamakhshary, Stress amongst dental students: a systematic review, Eur. J. Dent. Educ. 15(1) (2011) 8-18.

[8] K. Divaris, A. Polychronopoulou, K. Taoufik, C. Katsaros, T. Eliades, Stress and burnout in postgraduate dental education, Eur. J. Dent. Educ. 16(1) (2012) 35-42.

[9] A. Boran, M. Shawaheen, Y. Khader, Z. Amarin, V. Hill Rice, Work-related stress among health professionals in northern Jordan, Occup. Med. 62(2) (2012) 145-7.

[10] D. Bhugra, K.S. Bhui, K.R. Gupta, Burnout and stress among doctors and dentists in North India, Int. J. Cult. Ment. Health 1(1) (2008) 24-29.

[11] H.R. Chapman, S.Y. Chipchase, R. Bretherton, Understanding emotionally relevant situations in primary care dental practice: 1 . Clinical situations and emotional responses, Br. Dent. J. 219(8) (2015) 401-9; discussion 409.

[12] K.M.S. Ayers, W.M. Thomson, J.T. Newton, A.M. Rich, Job stressors of New Zealand dentists and their coping strategies, Occup. Med. 58(4) (2008) 275-281.

[13] R.E. Johns, D.M. Jepsen, Sources of occupational stress in NSW and ACT dentists, Aust. Dent. J 60(2) (2015) 182-9.

[14] E.J. Kay, J.C. Lowe, A survey of stress levels, self-perceived health and healthrelated behaviours of UK dental practitioners in 2005, Br. Dent. J. 204(11) (2008) E19; discussion 622-3.

[15] R. Moore, I. Brodsgaard, Dentists' perceived stress and its relation to perceptions about anxious patients, Community Dent. Oral Epidemiol. 29(1) (2001) 73-80.

[16] H.R. Chapman, S.Y. Chipchase, R. Bretherton, Understanding emotionally relevant situations in primary dental practice. 2. Reported effects of emotionally charged situations, Br. Dent. J. 219(9) (2015) E8-E8 1p.

[17] S.Y. Chipchase, H.R. Chapman, R. Bretherton, A study to explore if dentists' anxiety affects their clinical decision-making, Br. Dent. J. 222(4) (2017) 277-290.

[18] A. Plessas, M.B. Delgado, M. Nasser, Y. Hanoch, D.R. Moles, Impact of stress on dentists' clinical performance. A systematic review, Community Dent. Health 35(1) (2018) 9-15.

[19] J.M. Poolton, M.R. Wilson, N. Malhotra, K. Ngo, R.S.W. Masters, A comparison of evaluation, time pressure, and multitasking as stressors of psychomotor operative performance, Surgery 149(6) (2011) 776-782. 
[20] K. Moorthy, Y. Munz, A. Dosis, S. Bann, A. Darzi, The effect of stress-inducing conditions on the performance of a laparoscopic task, Surg. Endosc. 17(9) (2003) $1481-4$.

[21] S. Arora, N. Sevdalis, D. Nestel, M. Woloshynowych, A. Darzi, R. Kneebone, The impact of stress on surgical performance: A systematic review of the literature, Surgery 147(3) (2010) 318-330.e6.

[22] ALQahtani, J.I. Rotgans, S. Mamede, A.L. I, M.E. Magzoub, F.M. Altayeb, M.A. Mohamedani, H.G. Schmidt, Does Time Pressure Have a Negative Effect on Diagnostic Accuracy?, Acad. Med. 91(5) (2016) 710-6.

[23] E. Tsiga, E. Panagopoulou, N. Sevdalis, A. Montgomery, A. Benos, The influence of time pressure on adherence to guidelines in primary care: an experimental study, BMJ Open 3(4) (2013).

[24] M. Pearce, S.J. Agius, J. Macfarlane, N. Taylor, Supporting dental registrants in difficulty, Br. Dent. J. 218(4) (2015) E5.

[25] C.P. Hapcook, Sr., Dental malpractice claims: percentages and procedures, J. Am. Dent. Assoc. 137(10) (2006) 1444-5.

[26] T.K. Gandhi, A. Kachalia, E.J. Thomas, A.L. Puopolo, C. Yoon, T.A. Brennan, D.M. Studdert, Missed and delayed diagnoses in the ambulatory setting: a study of closed malpractice claims, Ann. Intern. Med. 145(7) (2006) 488-96.

[27] P.A. Mileman, E. Mulder, L. van der Weele, Factors influencing the likelihood of successful decisions to treat dentin caries from bitewing radiographs, Community Dent. Oral Epidemiol. 20(4) (1992) 175-180.

[28] P.A. Mileman, L.T. van der Weele, The role of caries recognition: treatment decisions from bitewing radiographs, Dentomaxillofac. Radiol. 25(5) (1996) 228-233. [29] A. Shelley, Reporting of dental radiographs in general dental practice, Prim. Dent. J. 2(1) (2013) 46-9.

[30] P. Mileman, L. van der Weele, F. van de Poel, D. Purdell-Lewis, Dutch dentists' decisions to take bitewing radiographs, Community Dent. Oral Epidemiol. 16(6) (1988) 368-373.

[31] L. Ordóñez, L. Benson, Decisions under Time Pressure: How Time Constraint Affects Risky Decision Making, Organ. Behav. Hum. Decis. Process. 71(2) (1997) 121140.

[32] D.G. Altman, J.M. Bland, How to randomise, BMJ 319(7211) (1999) 703.

[33] D.G. Altman, J.M. Bland, Diagnostic tests. 1: Sensitivity and specificity, BMJ 308(6943) (1994) 1552.

[34] J.D. Bader, D.A. Shugars, What do we know about how dentists make cariesrelated treatment decisions?, Community Dent. Oral Epidemiol. 25(1) (1997) 97-103. [35] C. Lucchiari, G. Pravettoni, Cognitive balanced model: a conceptual scheme of diagnostic decision making, J. Eval. Clin. Pract. 18(1) (2012) 82-8.

[36] G.R. Norman, K.W. Eva, Diagnostic error and clinical reasoning, Med. Educ. 44(1) (2010) 94-100.

[37] E. Zinman, Dental and legal considerations in periodontal therapy, Perio 200025 (2001) 114-130.

[38] H. Harvie, Periodontal Disease. Summons MDDUS Memb J. Spring 2007.14-15. [39] DDU. Probing deeper into periodontics claims. DDU J. March 2014:19-21.

[40] S.R. Hanney, M.A. Gonzalez-Block, M.J. Buxton, M. Kogan, The utilisation of health research in policy-making: concepts, examples and methods of assessment, Health. Res. Policy Syst. 1(1) (2003) 2.

[41] E.M. Kok, H. Jarodzka, Before your very eyes: the value and limitations of eye tracking in medical education, Med. Educ. 51(1) (2017) 114-122. 
Figures:

Figure. 1: Counterbalancing.

TP:Time Pressure, NTP No time pressure, A: Set A of radiographs, B: Set B of radiographs

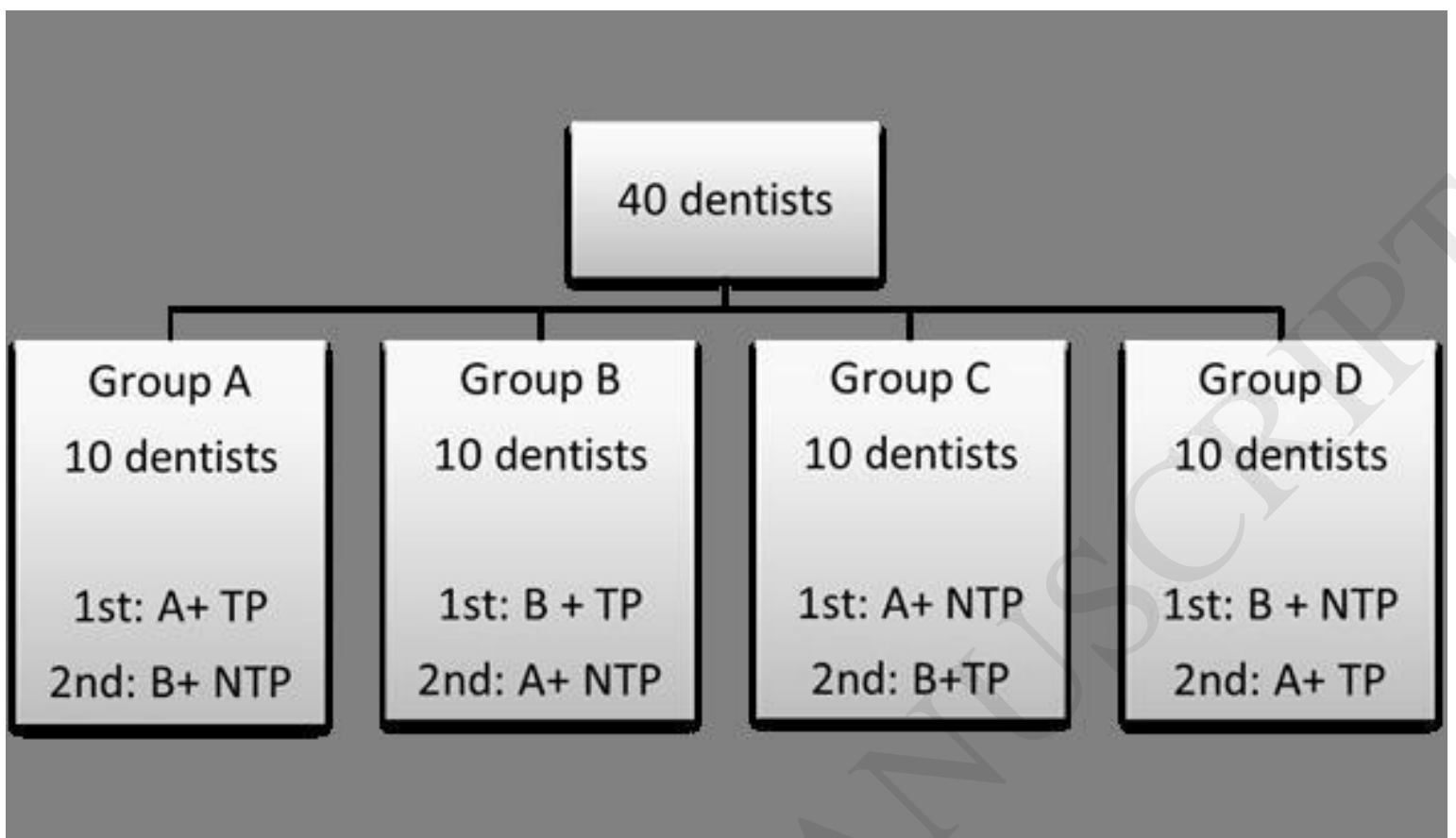


Figure 2: Delivery of the study

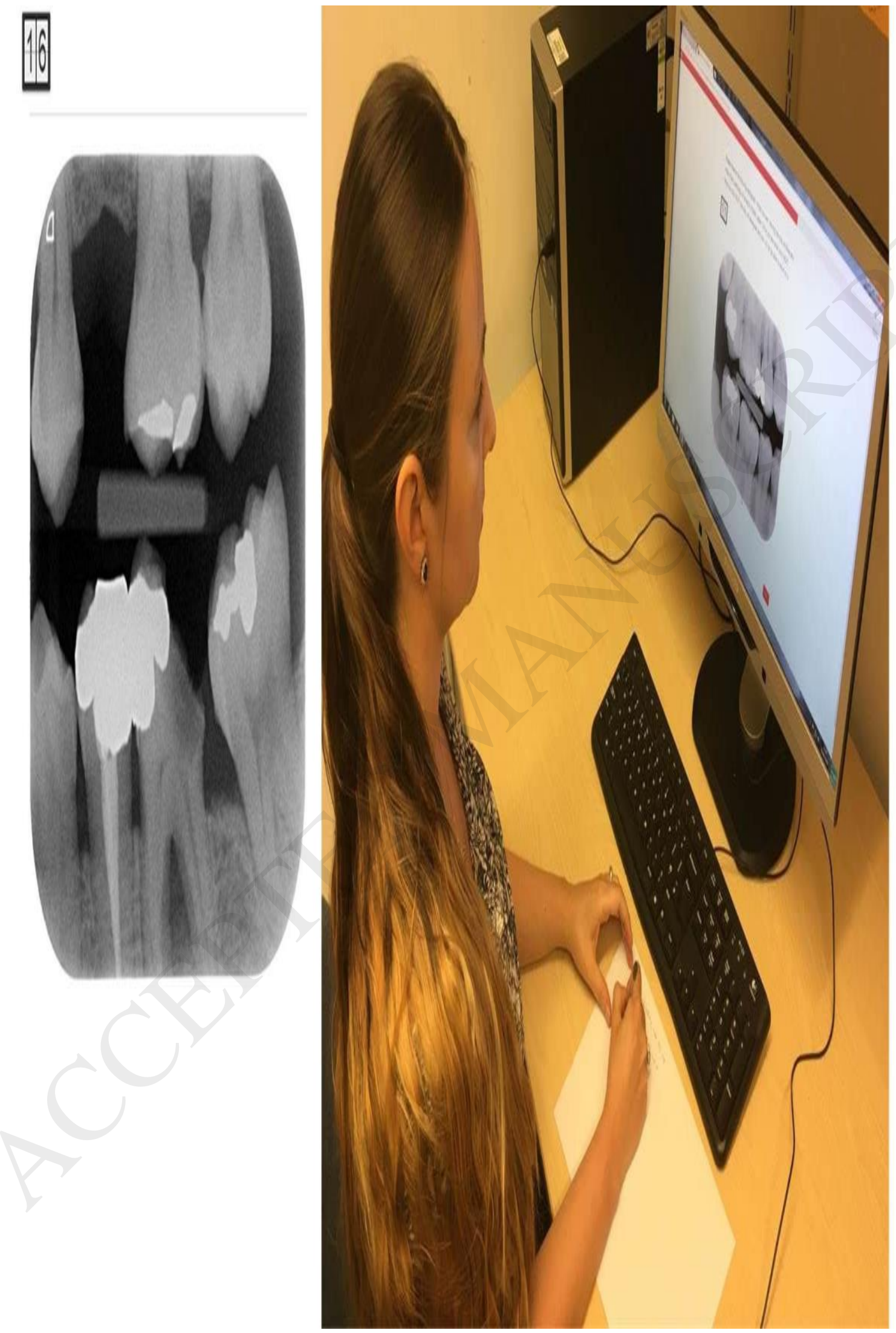


Figure 3: Electronic VAS scales used in the study. The marker can be moved across the scale $(0-100)$ to indicate the participants' stress level and level of difficulty they experienced under each condition.

How stressful did you find this task? Please use the scale of 0 to 100 below where 0 is not stressful at all and 100 is the worst imaginable level of stress.

not at all stressful

$0 \quad 10-20$

30

40

50

60

70

extremely stressful

80

$90 \quad 100$

How difficult did you find this task? Please use the scale of 0 to 100 below where 0 is no difficulty at all and 100 is the greatest level of difficulty imaginable.
not difficult at all
$0 \quad 10$
20
30
40
50
60
70
80
extremely difficult

Click to write Choice 1 


\section{Tables:}

Table 1: Reporting Radiographic Items

any radiolucency indicative of caries

any defective restorations

any overhangs of restorations

any bone loss

any angular bony defects

any furcation involvement lesions

any retained roots and

any impacted teeth 
Table 2: Participant characteristics

\begin{tabular}{|l|l|l|l|}
\hline \multicolumn{2}{|l|}{ Participant Characteristics } & Frequency (n) & $\begin{array}{l}\text { Percentage } \\
(\%)\end{array}$ \\
\hline \multirow{3}{*}{ Gender } & Male & 21 & 53.0 \\
\cline { 2 - 4 } & Female & 19 & 47.0 \\
\hline \multirow{3}{*}{ Type of Practice } & Private & 10 & 25.0 \\
\cline { 2 - 4 } & NHS & 10 & 25.0 \\
\cline { 2 - 4 } & Mixed & 13 & 32.5 \\
\cline { 2 - 4 } & Community & 7 & 17.5 \\
\hline \multirow{3}{*}{ Employment } & Full-time & 23 & 57.5 \\
\cline { 2 - 4 } & Part-time & 17 & 42.5 \\
\hline \multirow{2}{*}{ Postgraduate Qualifications } & Yes & 22 & 55.0 \\
\cline { 2 - 4 } & No & 18 & 45.0 \\
\hline
\end{tabular}


Table 3: Median Sensitivity and Specificity values for Set A radiographs under the two experimental conditions (time-pressure vs no time-pressure)

Table 3: Median Sensitivity and Specificity values for Set A radiographs under the two experimental conditions (time-pressure vs no time-pressure)

\begin{tabular}{|c|c|c|c|c|}
\hline \multirow{2}{*}{$\begin{array}{l}\text { Radiograph } \\
\text { (Set A) }\end{array}$} & Sensitivity & \multicolumn{3}{|c|}{ Specificity } \\
\hline & $P$ value & $\begin{array}{l}\text { Time } \\
\text { Pressure } \\
\text { (Median) }\end{array}$ & $\begin{array}{l}\text { No Time } \\
\text { Pressure } \\
\text { (Median) }\end{array}$ & P value \\
\hline \multirow[t]{3}{*}{$\mathbf{A 1}$} & $<0.0001$ & 1.00 & 1.00 & 0.076 \\
\hline & 0.006 & 1.00 & 1.00 & 0.231 \\
\hline & * & 1.00 & 1.00 & 0.799 \\
\hline \multirow[t]{3}{*}{ A2 } & $<0.0001$ & 1.00 & 1.00 & 0.156 \\
\hline & 0.429 & 1.00 & 1.00 & 1.000 \\
\hline & 0.183 & 1.00 & 1.00 & 0.602 \\
\hline \multirow[t]{3}{*}{ A3 } & 0.003 & 1.00 & 0.98 & 0.602 \\
\hline & 0.102 & 0.96 & 1.00 & 0.414 \\
\hline & 0.289 & 1.00 & 1.00 & 1.000 \\
\hline \multirow[t]{3}{*}{ A4 } & $<0.001$ & 0.99 & 1.00 & 0.904 \\
\hline & $<0.0001$ & 0.98 & 1.00 & 0.841 \\
\hline & * & 1.00 & 1.00 & 0.602 \\
\hline \multirow[t]{3}{*}{ A5 } & 0.012 & 1.00 & 1.00 & 0.495 \\
\hline & * & 0.94 & 0.94 & 0.799 \\
\hline & 0.183 & 1.00 & 1.00 & 1.00 \\
\hline \multirow[t]{3}{*}{ A6 } & 0.174 & 0.98 & 0.98 & 0.478 \\
\hline & 0.565 & 0.94 & 0.94 & 0.799 \\
\hline & 0.028 & 1.00 & 1.00 & 0.799 \\
\hline & \multicolumn{4}{|c|}{$\begin{array}{l}\text { Independent Samples Mann-Whitney U test } \\
{ }^{*} \text { The sensitivity could not be calculated for this radiograph as there was not caries/periodontal- } \\
\text { related pathology present }\end{array}$} \\
\hline
\end{tabular}


Table 4: Median Sensitivity and Specificity values for Set B radiographs under the two experimental conditions (time-pressure vs no time-pressure)

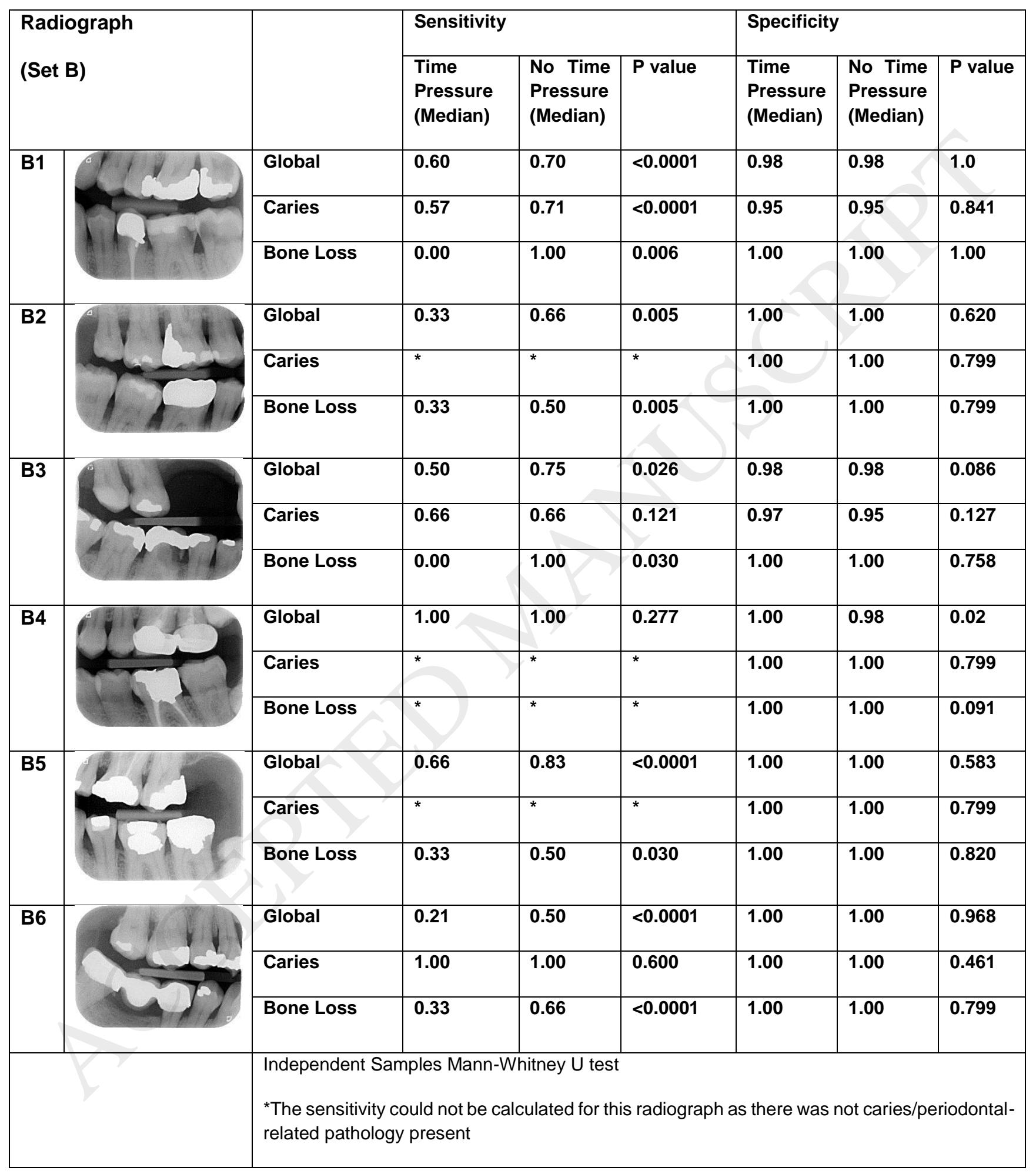

